https://doi.org/10.18778/2196-8403.2021.08

\title{
„Fangt an zu kochen - das Rezept folgt" (nach Brian Eno). Aber das Setting, die Zutaten, der Appetit und die Experimentierlust sollten schon gegeben sein. (Post-)dramaturgische und spiel- ästhetische Positionen und Diskurspotentiale
}

Die sich ästhetisch zunehmend durchdringende zeitgenössische Theater- und Performancepraxis weisen ein erhebliches Innovations- und Diskurspotential auf, das sowohl spielästhetische als auch (post-)dramaturgische Fragen auslöst. Dabei interessieren u.a. folgende Aspekte, gerade auch im Hinblick auf die künstlerische Ausbildungspraxis, besonders: Schein, Authentizität, Repräsentationskritik, Autorschaft, Stückentwicklung, (soziale) Teilhabe, Digitalisierung, Cross-gender-acting.

Schlüsselwörter: Schein, Authentizität, Repräsentationskritik, Stückentwicklung, Digitalisierung, Cross-gender-acting

"Start cooking - the recipe will follow". But the setting, the ingredients, the appetite and the wish to experiment should already be there. (Post-)dramatic aesthetic approaches and discourse opportunities

The overlapping forms of contemporary theatre and performance have created significant opportunities for innovation and new discourse which poses questions related to aesthetics and (post)dramatic concerns. How can we describe and characterize this artistic and theoretical knowledge? What are the main challenges, topics and strategies in artistic research and acting/performing? This essay is an attempt to map some essential fields in the (post-)dramatic landscape - including new forms of (post-) dramaturgical thinking and the shifting understanding of (collective) authorship. The following aspects are of particular interest, especially in relation to artistic training practice: appearance, authenticity, representational criticism, authorship, play development, (social) participation, digitization, cross-gender acting. 
Keywords: appearance, authenticity, critique of representation, Stückentwicklung, digitization, cross-gender acting

\begin{abstract}
„Zacznijcie gotować - a przepis przyjdzie“. Jednak sytuacja, przyprawy, apetyt i radość $z$ eksperymentowania powinny pojawić się wcześniej. Postawy (post-) dramatyczne oraz estetyczne i możliwości dyskursu

Nakładające się na siebie coraz częściej praktyki teatralne i performatywne odznaczają się dużym potencjałem innowacyjnym i dyskursywnym, prowokującym pytania dotyczące gry estetycznej jak i (post-)dramatyczności. Wyjątkowo interesujące są w tym przypadku, w szczególności w kontekście kształcenia artystów: pozór, autentyczność, krytyka ideologii reprezentacji, autorstwo, tworzenie sztuki teatralnej, partycypacja (społeczna), digitalizacja, Cross-gender-acting.
\end{abstract}

Słowa klucze: pozór, autentyczność, krytyka ideologii reprezentacji, tworzenie sztuki teatralnej, digitalizacja, Cross-gender-acting

Da schreibt man die Sätze im Voraus auf schön einen nach dem anderen damit der Sinn der Sache abgemacht ist ein abgekartetes Spiel das Schauspiel.

(REICHERT 2021:14)

\title{
Vielfalt/Verflechtungen
}

Schwerpunkte im zeitgenössischen Theater, allein im deutschsprachigen, pointiert zu bestimmen, fordert heraus. Das liegt an dessen vielfältigem sowie beweglichem künstlerischen Spektrum und den damit ins Spiel kommenden polyphonen Formen, Formaten, Spielweisen und freigesetzten Narrativen. Aber ebenso daran, dass auch im Theater, relationale Ästhetiken' eine immer wichtigere Rolle spielen und entsprechend miteinander verzweigte, kunstformende sowie medienübergreifende Diskursschauplätze und Formen anstiften. Diese rufen nicht nur multiperspektivische ästhetische Ereignisse hervor, sondern versuchen gerade auch soziale Situationen in künstlerisch gerahmten Räumen zu begründen (vgl. BOURRIAUd 1998). Gewichtige künstlerische Positionen setzten sich somit, wie ich betonen möchte, verstärkt aus verschiedenen, miteinander spielenden inhaltlichen Setzungen und formalen Zugriffen zusammen und legen dabei zunehmend reflexiv ihre eigenen Strukturen frei. Wie lassen sich zunächst die für mich zentralen Positionen trotz ihrer Heterogenität auf einen Minimal-Nenner bringen - ohne diese bloß additiv zu katalogisieren? Dazu einige unter Stichwörtern von mir versammelte Gedanken. 


\section{Repräsentationskritik}

Ein wesentlicher Ausgangspunkt sind für mich repräsentationskritische szenische Versuchsanordnungen, die grundsätzlich jeweils spürbar hohe performative Energien anstreben und einen entscheidenden Schritt vollzogen haben: Es geht heute - und damit meine ich gleichermaßen Theatertexte wie die Aufführungspraxis - längst nicht mehr um das bloße Formulieren von Repräsentationsskepsis, sondern um das bewusste performative Durchstreichen von überholten Repräsentationsmustern, das in einer im Vollzug begriffenen (Teil-)Dekonstruktion dieser mündet.

Mais attention! Die Repräsentationsdiskurse, die auch aufgrund ihrer (post-) ideologischen Aufladungen z.T. ein erhebliches ästhetisch-politisches Potential aufweisen können, dauern an. Warum das nicht nur gut, sondern elementar ist, zeigt die Praxis des Schauspiels.

Anders formuliert: Zeitgenössische Aufführungen im Schauspiel halten selbstbewusst an der Praxis der Repräsentation fest - was in ihre künstlerische DNA eingeschrieben ist - denken aber verstärkt auf den miteinander verschränkten strukturellen sowie ästhetischen Ebenen aktiv Repräsentationskritik mit.

Repräsentation ist allein schon deswegen nicht von der Bühne wegzudenken, da diese zwei Bedeutungen entfaltet, die nicht voneinander zu trennen sind: die des Stellvertretens und die des Darstellens:

Wird im Theater die Differenz zwischen Repräsentation und Repräsentiertem nicht (ganz) aufgehoben, werden Kunst und Leben nicht eins, was bedeutet, dass nach Brechts Konzept des gestischen Spiels - also des verweisenden Zeigens - die Stellvertretung der Bühnenfigur durch den Schauspieler*in in ihrer Symbolik sichtbar bleibt. (MALZACHER 2020:29)

Dieser Zeigegestus, so FLORIAN MALZACHER (2020:29) weiter, verweist wiederrum auf ein doppeltes, paradoxal anmutendes Moment, an dem sich das Theater kontinuierlich reibt: „die Unmöglichkeit der totalen Repräsentation als auch die Unmöglichkeit einer totalen Nicht-Repräsentation“. Und genau das macht einen zentralen Reiz des Theaters aus.

Ich gehe mit JAKOB HAYNER (2020:108) erstens davon aus, dass ,eine Repräsentationskritik, die nur auf die bloße Unmöglichkeit oder schlichte Vervielfältigung des zu Repräsentierenden setzt, ohne ins Zentrum der künstlerischen Darstellung von Wirklichkeit vorzudringen, ihren Zweck verfehlt.“

Und zweitens mit dem Kollektiv Gob Squad, die wohl die wichtigste Herausforderung einer zeitgemäßen Repräsentationskritik auf den Punkt bringen: 
„Wie vermeiden wir, das zu reproduzieren, was wir eigentlich kritisieren?“ (SQUAD 2020:60). Die Frage von Gob Squad klingt eigentlich selbstverständlich, ist aber weiterhin herausfordernd, da hier nicht nur ein politischer Anspruch im ästhetischen Diskurs durchklingt, sondern auch die strukturelle Dimension in Form der eigenen Arbeitsweise - und somit auch ein soziales Moment - angesprochen wird.

Eine Schwerpunktverschiebung, die heute immer mehr in den Fokus der Aufmerksamkeit gerät, da der direkte Transport von künstlerisch verdichteten Inhalten seitens der darstellenden Künste zunehmend mit der Fokussierung auf deren prozessartiger sowie widerständiger Hervorbringung konkurriert. So drücken sich Probenprozesse, vor allem in ihrer kollektiven Dimension, verstärkt in Aufführungen ab oder werden in diesen offensiv thematisiert. Damit meine ich speziell die betont situative oder improvisierte Text-/Szenengenerierung in Aufführungen in Form von transparenten ,Making-of-Ästhetiken“

„Theater ist Erfahrung, keine Mitteilungsform“, so auch Heiner Goebbels (zit. nach HAYNER 2020:33).

Dies führt u.a. dazu, dass Aufführungen dazu neigen, offener, durchlässiger, brüchiger und dadurch tendenziell diskursiver zu sein. Auch dadurch, dass kontinuierliche dramatische Spannungsbögen durch den Abbau von nicht handlungsbasierenden Narrationen oder psychologisierende Muster bewusst verweigert werden und stattdessen mit Störungs- und Zerstreuungsmomenten operiert wird, welche die Wahrnehmung der Schauspieler*innen sowie des Publikums immer wieder verschieben. Damit wachsen zugleich der potentielle Selbstreflexivitätsgrad, das ,Live-Denken“ sowie das mediale Bewusstsein der beteiligten Akteur*innen an. Aber auch die Gefahr, dass die potenzierte Selbstbezüglichkeit, sich in sich selbst erschöpft und die Formate schrumpfen lässt.

\section{Mehr Kollektivität und Teilhabe. Ästhetik und Struktur(en)}

Es war und ist offensichtlich - obwohl das Theater von seiner Grundidee her doch gerade eine kollektive Kunstform ist (vgl. STEMANN 2019:64): „Es gibt eine Führungskrise der über Jahrzehnte eingeübten Machtstrukturen im Theater. Diese Strukturen sitzen uns allen so tief in den Knochen, dass man auch selbst nicht davon gefeit ist, diese zu reproduzieren - egal ob man ein Mann oder eine Frau ist", so die Karlsruher Schauspielchefin Anna Bergmann (zit. nach KÜMMEL 2020:57). 
Vielleicht zeigt gerade der Blick nach Zürich, dass sich der Wind dreht: kollektivere künstlerische Arbeitsformen mittels flacherer Hierarchien, größere künstlerische Gleichberechtigung, Teilhabe und Offenheit, aber auch, so Stemann, „weniger mit mehr Leuten zu machen“, ein stärkeres „Andocken der freien Szene“ sowie eine intensivierte Arbeit mit Jugendlichen" (STEMANN 2019:70).

Als Vorbild fungieren hier elastischere Strukturen aus der „freien Szene“, die Amelie Deufelhard jüngst wie folgt skizzierte: „Die flexiblen, kollektiven, spartenübergreifenden Arbeitsweisen versetzen gerade die Performance-Szene in die Lage, Visionen und Projekte für die Krisenbewältigung zu entwickeln, auch weil interdisziplinäres Arbeiten in sie eingeschrieben ist“" (DEUFELHARD 2020:47).

Ich spreche dieses strukturelle Moment vor allem aus einem Grund an. Avancierte formbewusste und sich politisch artikulierende Spielästhetiken fordern vehement alternative Arbeitsweisen bzw. andere Probenbedingungen ein. Eine Herausforderung auch für die künstlerische Ausbildungspraxis.

Und zwar, dass diese sowohl konkret auf die Veränderungen reagiert als auch selbst agiert, indem sie unter ,geschützten Laborbedingungen“ als ebenso schöpferischer wie kritischer Impulsgeber zukünftige Spiel- und Darstellungsformen mitpräfiguriert.

Dabei erscheint es umso wichtiger, die Folgen der sich ändernden künstlerischen Arbeitsstrukturen, d.h. gerade auch die ,projektzentrierten' spartenübergreifenden Arbeitsweisen der ,freien Szene', in der Ausbildungspraxis gemeinsam mit den Studierenden kritisch zu diskutieren und dabei ebenso kontinuierlich die eigenen Ausbildungsstrukturen zu spiegeln. Speziell mit dem Ziel, die künstlerische und politische Mündigkeit der Absolvent*innen, die ein ausgeprägtes Selbstbewusstsein voraussetzt, maximal zu fördern.

\section{Schauspiel/Performance}

Mit den gegenwärtigen strukturellen Veränderungen spielt noch ein weiteres, d.h. ästhetisches Moment zusammen: die sich intensivierende Befruchtung und ,ästhetische Durchdringung der Schauspiel- und Performancepraxis', die sich zunehmend in ästhetisch ,hybriden Verschmelzungsformen' äußert und ebenso Ästhetiken des Films, der bildenden Künste und des Tanz- und Musiktheaters berührt. Zunächst aber eine wie eine Gegenposition erscheinende Diagnose von Helmut Schäfer, der die wesentlichen Differenzmerkmale zwischen Schauspieler und Performer markiert und auf ein potentielles spielerisches Verarmungsmoment hinweist, das gravierende Folgen mit sich bringen kann: 
Was der Schauspieler grundsätzlich aufsucht, ist der Zweifel an den Bekundungen der Figur. Auch in Bezug auf ihre Motive. Die tieferen Gründe der Figur erschließen sich ihm um so mehr, wie er ihr Begehren, ihre Abgründe oder Verdrängungen durchdringt. Diese Suche nach den verborgenen Motiven und Antrieben der Figur verhindert, daß der Schauspieler in eindeutige Aussagen übersetzt. Das aber versucht der Performer. Der Performer ist der vom Theater der Psychologie des Bürgertums abgewiesene Schauspieler. Wenn sich im Performer, der im Gegensatz zum Schauspieler nur für sich selbst steht, die Idee des Individuums in der bürgerlichen Gesellschaft vollendet, schließt das Schauspielertheater an die Erfahrung des Ensembles wie auch die der Zuschauer an. Wer dagegen als er selbst auf der Bühne steht, begreift Realität als eine wenn auch mehrdeutige, so doch identifizierbare Wirklichkeit und hat größere Schwierigkeiten, ein Anderes zu realisieren, was ein Ensemble von Schauspielern als Organismus explizit aufzufinden bemüht. (SCHÄFER 2020:94)

Und dennoch, - es geht in der Praxis längst nicht mehr (nur) um die ,ästhetische Bipolarität‘ von Schauspiel und Performance bzw. Schauspieler*in und Performer*in und die Frage des jeweiligen Innovationspotentials.

Martin Wuttke bezeichnete sich unlängst in der Pollesch-Inszenierung „(Life on earth can be sweet) Donna" (Deutsches Theater Berlin 2019), d.h. in einer an Brechts ,Straßenszene“ angelegten Schauspiel-Performance mit ausuferndem ,Rollenspiel“ (!) augenzwinkernd als „Transformer“.

Ob Performer*in oder/und Schauspieler*in, relevant ist, wie sich beide im Sinne von Fabian Hinrichs als Künstler*innen von ,Servicekräften“ in Dienstleistungsbetrieben absetzen, d.h. künstlerisch/szenisch widerständig agieren, eigenverantwortlich und offensiv die Eigengesetzlichkeiten ihres Mediums hinterfragen, im Miteinander Menschenbilder verhandeln und weiterhin Weltentwürfe zu formulieren wagen (vgl. HINRICHS 2018). Und dabei auch das in der Gesellschaft anhaltende Authentizitätsdoktrin „Sei Du selbst“ entzaubern - und gerade deshalb ,spielerisch wahrhaftig' auf- bzw. hervortreten.

Oder zugespitzt mit Devid Striesow: „Authentizität, das gibt es nicht. Das lernt man in der Schauspielschule“(zit. nach ENGLER 2017:7).

Stimmt und stimmt nicht, je nach Perspektive, da es (auch auf der Bühne) sowohl darum geht, „man selbst“ zu werden, ,indem man in Beziehung zu sich tritt“ als auch darum, sein ,Selbst“ spielerisch zu bewegen und (neu) zu formen oder zu verformen (ENGLER 2017:146). Der zweite Vorgang fordert wiederrum auch ein Abstandnehmen von sich selbst bzw. ein auf sich Heraufschauen ein, das Engler als „Differenz“ bezeichnet: „Menschen sind nie näher bei sich, au- 
thentischer, als in jenen Momenten, in denen sie aus der Differenz zu sich heraus zu spielen anfangen“" (ENGLER 2017:130). ${ }^{1}$

\section{Digitalisierung}

Wie verhält sich das Theater zur voranschreitenden Digitalisierung? Muss das Theater zu einem ,Digitalisierungstheater' werden, um zeitgemäß zu sein?

Ich denke nicht, da die zentralen Theaterformen nach wie vor ihre Kraft durch die reale Präsenz der Schauspieler*innen und die reale Anwesenheit des Publikums in einem nicht virtuellen Raum entfalten und dabei, so die entscheidende Qualität, gemeinsame“ Erfahrungen generieren - vor allem unmittelbare körperliche Erfahrungen.

Aber die sich auftuenden neuen - und durch das Coronavirus zusätzlich beschleunigten - potentiellen Spielräume im Digitalen und nun wieder verstärkt mit dem Digitalen werden immer sichtbarer und laden dazu ein, über neue Formen und Formate nachzudenken.

Die Herausforderung besteht hier vor allem darin, möglichst produktiv/kreativ nachhaltige Fragen zu generieren anstatt reflexhaft vorschnelle Antworten zu formulieren, die lediglich eine ästhetisch digital-hybride (Theater-)Kunst auf der ,Höhe der Zeit‘ suggerieren. ${ }^{2}$

1 Engler schränkt ein und präzisiert: „Selbstverständlich spielen Kinder, Erwachsene, professionelle Spieler stets auch um des Spielens, um jener Freiheiten, jener Möglichkeiten willen, die das Spiel gewährt. Erwartungen, Regeln der realen Welt in der eingebildeten Kraft außer Kraft zu setzen, Realitätsaneignung mittels Realitätsüberschreitung, darin liegt der Witz, der Reiz des Spiels. Spielen ist ein ambivalenter Vorgang, der besonders Kinder oftmals irritiert. Sie erfinden eine Welt, verhalten sich zu dieser mit demselben Ernst, als wäre sie die außerspielerischreale, klagen Absprachen, Regeln ein, und fallen, indem sie den Selbstbetrug bemerken, ins Lachen, verlachen ihren Ernst und kommen doch nicht davon los“ (ENGLER 2017:130f.).

2 Dass in diesem Kontext anstatt schneller Antwortbemühungen zunächst das offene Aushandeln von ausdifferenzierten Fragen und deren spielerisch-experimentelle Befragung im Mittelpunkt stehen, betonen ebenso Birgit Wiens, Bettina Milz oder Judith Ackermann sowie Benjamin Egger mit unterschiedlichen Akzentuierungen (vgl. ZELLNER / LOBBES / ZIPF 2021). 
Es ist offensichtlich, dass in naher Zukunft neue digitale Sparten bzw. digitale Spielstätten an größeren oder kleineren Häusern selbstverständlich sein werden. ${ }^{3}$ Also eine Kopräsenz von Theater (offline) und interaktivem Netztheater (online), wobei letztere, gerade im Hinblick auf ihre (Gaming-)Dramaturgien, die Aufmerksamkeit auf die ,digitale Narration' in Form eines ,vernetzten Erzählens', das wiederrum verstärkt einen seriellen Charakter aufweisen könnte, lenken werden (vgl. LOBBES / SIMON 2020: 64). Ihre Nachhaltigkeit wird entscheidend davon abhängen, ob die „Gestaltung von Vergemeinschaftung als eine der größten Herausforderungen" (KIRSCHNER 2020:73) gelingt. Folgende zentrale Voraussetzung dafür erscheint mir höchst plausibel: „Aber die Antworten auf die Frage nach der Gestaltung unseres neuen Miteinanders werden wir nicht in einer Übertragung vergangener künstlerischer Formate finden“" (KIRSCHNER 2020:73).

Caren Jess lenkt wiederrum den Fokus auf das Theater und nennt einen Aspekt, der mir besonders dringlich erscheint: „Während das Digitale aufzeigen konnte, was das Theater nicht kann, sollte das Theater zeigen, was das Digitale nicht kann. Nicht um Formen gegeneinander auszuspielen, sondern den jeweiligen Eigenwert unter Beweis zu stellen“ (JESS 2020:53).

Geht es denn letztlich nicht beim Zusammentreffen des analogen und digitalen Theaters um die entscheidende Frage, , wer hier wen inszeniert'? D.h., , das Theater die Technologie?' Oder, wie Christoph Gürk befürchtet, „die Technologie das Theater?" (GÜRK 2020:17). Kann die Motivation für die Theater eigentlich größer sein, als Gürks Befürchtungen von den realen Bühnen zu fegen?

Thomas Ostermeier fragte sich hingegen jüngst: „Müssen wir uns mehr auf die einfachen Formen des Theaters, das Spielerische, die Verwandlung besinnen?“ (OSTERMEIER 2020:53). Ich teile seine Haltung und empfinde diese keineswegs als rückwärtsgewandt. Sie ist aber eine Option von mehreren.

3 Ein Beispiel wäre hier die von Kay Voges 2019 gegründete Dortmunder Akademie für Theater und Digitalität, die sich in ihrer Selbstwahrnehmung - zumindest bis jetzt - nicht schwerpunktmäßig als ,produzierender Betrieb 'versteht. Primär produziert wird dagegen aktuell u.a. in der Online-Sparte HAU 4 des Berliner HAU oder am Staatstheater Augsburg, das wiederrum mit der Dortmunder Akademie für Theater und Digitalität im Rahmen des gemeinsam begründeten theaternetzwerk.digital kooperiert bzw. mit Formen des Digitaltheaters und der Theaterdigitalisierung experimentiert. 


\section{Der Schein trügt (nicht)}

Folgenreich ist, dass Ostermeier neben den zwei Kernmerkmalen indirekt noch eine weitere zentrale ästhetische Kategorie des Theaters ins Spiel bringt: den Schein. Der Schein hat es jedoch speziell im Hinblick auf aktuelle gewichtige dokumentarisch geprägte, also authentifizierende Theaterformen, nicht leicht. Grund dafür ist, dass diese prinzipiell an dessen Auflösung und damit auch am Abbau der Phantasie des Publikums arbeiten. Und zwar zu Gunsten harter Realitäten, die gerade dann an (Diskurs-)Kraft verlieren, wenn diese lediglich (als readymades) szenisch installiert werden.

Konträr dazu bezeichnet der Dramatiker Wolfram Lotz „die Fiktion als einzig schützenswerte Realität des Theaters“ (zit. nach DECKER 2020:85).

Der Schein hat entscheidende Qualitäten und bietet dem Schauspiel viele Spielmöglichkeiten:

Das Spiel lässt das spielende Subjekt mutieren. Wenn man das Spiel preisgibt, [...] gibt man das Wesen des Theaters preis, das gerade aus dem Spiel, also dem Schein des vermutlich Wahren seine emanzipatorische Kraft bezieht. [...] In dem Moment, indem ich die Kraft der Phantasie auflöse, um Präsentiertes durch Wirklichkeit zu beglaubigen, wird verhindert, dass die Einbildungskraft die Realität übersteigt und ihre Beschränktheit erkennt oder die Art und Weise ihrer Verfasstheit erfahrbar macht. (SCHÄFER 2020:93f.)

Helmut Schäfer weist noch auf eine weitere elementare Erfahrung hin, die deutlich macht, wie Realitätserfahrungen aus dem Schein hervor-/ausbrechen können:

Wohne ich auf einer Probe oder im Theater als Zuschauer dem Verhalten zweier Schauspieler bei, das ich durch den Versuch des Verstehens nicht unmittelbar auflösen kann, erzeugt dieser Vorgang in mir ein Staunen und wirft zugleich Fragen nach dem Wesen der Realität auf. (SCHÄFER 2020:95)

\section{Neue Beziehungsmuster: Schauspieler, Figur, Rolle und Diversität}

In den letzten Jahren hat sich die Relation von Schauspieler und Figur spürbar verändert, was sich in auffällig heterogenen Schauspielästhetiken und in einer sich stark veränderten (post-)dramaturgischen Praxis ausdrückt. Dazu fünf für mich relevante Positionen:

1. Franz WiLle in Bezug auf die Theaterästhetik von JÜRGEN GosCH: Nicht der Schauspieler verwandelt sich in eine Figur, sondern er/sie verwandelt seine/ihre Figur zu sich. Dabei wird nicht das Schauspieler-Sein komplett in die dramatische Fiktion gekippt [...], sondern die Fiktion des 
Stücks wird soweit und solange ins probengewandte Sein des Schauspielers herübergezogen, bis die Gegenwart in ihm rebelliert (WILLE 2006:114).

2. JOHAN SIMONS: Theater vermittelt sich über Gedanken, nicht über Identifikation. Die Schauspieler*innen sollten nicht nur versuchen, die Rolle zu spielen, sondern auch die Gedankenwelt des Hier und Jetzt zu zeigen. Man entkommt dem ohnehin nicht, sich selbst zu beobachten und zu reflektieren. Als Schauspieler*in sollte man sich nicht darum bemühen, die ganze Zeit mit der Figur zu verschmelzen. [...] Das Besondere am Schauspiel ist, dass man sich mit Problemen identifizieren kann. Mit Gedanken. (SIMONS 2018:15, 18 / SIMONS 2020:71)

3. LEONIE BÖHM: Ich versuche mit meinem Theater an Formen des Zusammenseins oder Kommunikationsformen zu forschen, die offen und gewaltfrei sind, und die nach der persönlichen Freiheit im Hier und Jetzt mit dem Anderen suchen. Ich will unbedingt, dass die Spieler*innen ein Gefühl für ihre Autorschaft bekommen. (zit. nach KLAEUI 2020:105)

4. YAEL RONEN: Eine sehr minimalistische Spielweise, die sehr verwirrend sein kann mit schwer fassbaren Unterschieden zwischen Figur und authentischer Reaktion. Die Schauspieler erschaffen parodistische Versionen ihrer selbst, aber auch anderer Meinungen, die sie repräsentieren. Sie müssen also Figuren spielen, die ihre Namen tragen, aber nicht unbedingt sie selbst sind. (WILLE 2019:104)

5. ANTA HelenA ReCKE: Ich möchte in den Bildern, die ich in die Welt setzte, grundsätzlich nicht die Illusion weiterführen, dass wir in einer weißen Welt leben. Deswegen kann es für mich kein Stück geben, in dem nur weiße Menschen gezeigt werden. Ich glaube, meine Arbeit wird sehr stark aus präzisen, abstrakten und theoretischen Ideen generiert. Wenn man das benennen müsste, in welchem Feld ich arbeite, dann würde ich am ehesten sagen, dass es um das Feld der Wahrnehmung geht. (RECKE 2019)

Heute genügt es weder, dass die Schauspieler*innen mit der Rolle noch mit ihrem performativen Selbst identisch sind. Gefragt sind Zwischenzonen oder Konfigurationsprozesse, d.h. Spiel- und Handlungsräume, in denen die Schauspieler*innen im Dialog mit ihren Figuren (noch) mehr Verantwortung für ihre persönliche spielerische Präsenz übernehmen und dabei eine gesteigerte Autor$s c h$ aft anstreben, die sich szenisch u.a. als lautes/hörbares Denken unterschiedlich äußern kann. Die Autorschaft fordert jedoch von allen Beteiligten ein gewachsenes kollektives dramaturgisches Bewusstsein, Denken und Wissen ein, das sich zunehmend gegen eine von der Regie und Dramaturgie bestimmte zen- 
tralperspektivische Wahrnehmungsordnung richtet und stattdessen zwischen allen Beteiligten diskursiv zirkuliert. Voraussetzung dafür ist ebenso ein feines szenisches Bewusstsein aller spielerisch handelnden Akteur*innen.

Dabei sind die Spielanlässe gemäß der Spielvorlagen höchst divers. Stücke/Theatertexte können vorliegen - ob als klassische oder zeitgenössische Dramen, Klassiker-Über-Schreibungen, Sekundärdramen, Roman- oder Filmadaptionen etc. Oder nicht vorliegen, wie bei Stückentwicklungen, die zumeist kollektiven /kollaborativen Rechercheprojekten entspringen.

Letztere neigen dazu, dass Schauspieler*innen persönliche Erfahrungen in Form von biographischen oder auto-fiktionalen Splittern in die Arbeit einfließen lassen, was wiederrum bei der szenischen Organisation des Materials eine ebenso offene wie kollektive Dramaturgie einfordert, d.h. eine Art Post-Dramaturgie bzw. eine Dramaturgie ohne Drama (vgl. UMATHUM / DECK 2020).

So z. B in den Stückentwicklungen von YAEL RONEN, die mittels intersektionaler Strategien gender-, race- und class-Diskurse kurzschließt, um vorherrschende singuläre Deutungshoheiten mit unterschiedlichen (postmigrantischen) Blickwinkeln zu dekonstruieren und dabei eigene „,blinde Flecken“ und Widersprüche im/über das Ensemble sichtbar zu machen, in denen sich das Publikum spiegeln kann. Hier liegt im Idealfall ihr politisches Potential. ${ }^{4}$

Einen anderen Ansatz stellt ANTA HELENA RECKES Schwarzkopie mit afrodeutschen Schauspieler*innen in Anna-Sophia Mahlers Inszenierung/Romanadaption Mittelreich von Josef Bierbichler dar.

Eine für das Schauspiel folgenreiche ästhetische Zäsur mit einem unmittelbar sichtbaren starken politischen Impetus in Form einer repräsentationskritischen postkolonialen szenischen Versuchsanordnung, die weitreichende Fragen aufwirft. Fragen, die Dekonstruktionsstrategien des eurozentristischen Blickregimes und damit verbundene strukturelle Rassismusmuster ebenso betreffen, wie das für die Appropriation Art spezifische Verhältnis von Original und Kopie, Wiederholung und Differenz (vgl. MÜLLER-SCHÖLL 2020:209-230).

JOHAN SIMONS diagnostiziert in Bezug auf seine Hamlet-Inszenierung, dass im Theater eine Frau einen Mann, ein Junger einen Alten, eine Schwarze eine Weiße

4 Dass sich hier die Kunst verstärkt gegenüber identitätspolitischen Positionen verhalten und selbst signifikant positionieren muss, die einen zunehmend erhitzten bzw. diskursiv aufgeladenen zentralen Schauplatz (der Sprache) markieren, reflektiert aus französischem Blickwinkel exemplarisch Carole FOUREST (vgl. 2020) in ihrer kontroversen Kritik an linken identitären Positionen. 
spielen kann, aber erst in dem Moment, indem man sich nicht mehr fragen würde, warum eine schwarze Schauspielerin, Mercy Dorcas Otieno, Königin Gertrud spielt und was das bedeuten soll, die Zeit der Diskriminierung endlich vorbei sei (vgl. SIMONS 2019). SIMONS präzisiert: „Mit meiner Besetzung mache ich keine politischen oder feministischen Statements, sie ist rein künstlerischer Natur" (SIMONS 2019).

Allerdings habe ich einige Zweifel, ob sich diese Ebenen nicht zunehmend/zwangsläufig durchdringen. Anders gefragt: Setzen diese ästhetische Setzung und dramaturgische Entscheidung - die auch die Besetzungen von Sandra Hüller als Hamlet und Gina Haller als Ophelia/Horatio betreffen - nicht einen politisch/feministisch mitgeprägten Diskurs voraus als auch frei, der zwangsläufig bereits im kollektiven künstlerischen Arbeitsprozess wirkt? Eine für mich grundsätzliche Frage mit viel Sprengkraft, die unmittelbar das künstlerische/politische Selbstverständnis berührt und, wie ich finde, auch im (kunst-)universitären Rahmen intensiv zu diskutieren ist.

\section{Räuberinnen, Volksfeindinnen, Platonowa und mehr. Cross-gender acting}

Dass Frauen immer häufiger Männerrollen spielen, ist weniger ein Trend als ein ,Paradigmenwechsel' im Theater, der gesellschaftliche Transformationsprozesse spiegelt und auf diese reagiert: Geschlechternormen ändern, diversifizieren sich, ihre Zuordnungen werden immer fluider (vgl. KASCH 2020).

Diese Rollenwechsel stellen aber vor allem auch einen männlich bestimmten Kanon im Theater selbst in Frage, der sich sowohl auf der Ebene der Darstellung als auch der Autorschaft in Form eines anhaltenden Ungleichgewichtes ausdrückt. Wie versucht also das Schauspiel überholte, speziell weibliche Rollenmuster und verkrustete Geschlechterkonstruktionen zu überwinden? Und wie lassen sich Weiblichkeit und Männlichkeit als gleichwertige und ggf. auch austauschbare Kategorien szenisch etablieren?

Einen Impuls setzte hier u.a. die Regisseurin SUSANNE ZAUN mit ihrer 2019 am Schlosstheater Moers uraufgeführten Stückentwicklung Die Mutter aller Fragen oder 25 Rollen, die eine Frau niemals spielen sollte. Bereits der Ankündigungstext betont die Dringlichkeit des Anliegens:

Was haben Ophelia, Julia, Louise, Marie, Gretchen, Käthchen, Lulu und Judith gemeinsam? Sie alle opfern sich seit Jahrhunderten für die Liebe und sterben vor unser aller Augen beeindruckende Bühnentode. Sie stammen aus Welten, die von 
männlichen Autoren entworfen wurden, ihr Handlungsspielraum wird von männlicher Macht bestimmt. Noch immer dominieren die Klassiker die Spielpläne der Theater, noch immer herrscht auch dort strukturelle Ungleichheit der Geschlechter. Höchste Zeit, genauer nachzufragen. Wie ist es, in jeder Vorstellung zu sterben, während der männliche Kollege wieder vorne an der Rampe steht und spricht? (SCHLOSSTHEATER MOERS 2020)

So geht es darum, weibliche Figuren anders zu gewichten, diese durch größere Handlungsspielräume aus ihrer eher passiven Opferrolle zu befreien, Konflikte und geschlechtliche Machtverhältnisse neu zu definieren, Blickwinkel zu verschieben und anders, d.h. in neuen oder umgekehrten geschlechtlichen Konstellationen zu spielen.

Mehr weibliche kritische Hamlets, Lears oder Baals. Mehr zeitgenössische ,Klassiker-Überschreibungen', die insbesondere Fragen nach der Rolle, Bedeutung und Inszenierung von Geschlecht durch möglichst spielintensive Rollenwechsel fokussieren.

Es ist zu erwarten, dass Dramatiker*innen, aber ebenso Schauspiel- und Regiestudierende, speziell in Form von Stückentwicklungen, weitere, daran anknüpfende Ansätze und neue Formate entwickeln werden. Auch hier gibt es - ebenso in dramaturgischer Hinsicht und in Bezug auf die Frage der Autorschaft der Schauspieler*innen - viel zu tun.

Mit ,Hosenrollen ' werden schon sehr bald ganz andere Assoziationen verbunden sein!

\section{Dramaturgische Spielräume}

Theoretisch zu denken heißt für mich in der künstlerischen Ausbildungspraxis und im Rahmen von artistic research gerade auch dramaturgisch zu denken und praktisches dramaturgisches Denken fordert wiederrum eine hohe Sensibilität gegenüber künstlerischen Prozessen und damit auch ein künstlerisches Denken ein. Was ist nun das Künstlerische an der dramaturgischen Praxis?

1. Dramaturgisches Denken verlangt heute u.a. eine potenzierte Medienkompetenz. Eine Medienmündigkeit, die ebenso für Schauspieler*innen in Form eines kunstformenübergreifenden medialen Bewusstseins und Wissens elementar ist und zunehmend die diskursiven (post-)digitalen Spielräume miteinschließt. Ein mediales Bewusstsein, das über den Rahmen des Theaters hinausgeht ohne diesen zu vergessen, befeuert szenisches und damit auch dramaturgisches Denken. 
Eine immer wichtigere Rolle spielt auch die soziale Kompetenz und Phantasie, die wiederrum ein kommunikatives Miteinander sowie eine Milieusensibilität voraussetzen, das mir ebenso in meiner Lehre viel bedeutet. Damit komme ich zu meinem zweiten Punkt.

2. Ich verstehe Dramaturgie, und ich verzichte hier einmal bewusst auf das Präfix Post, vor allem als kollektive Praxis und kollektive diskursive Hervorbringung (vgl. MAAR 2020:165-181) - oder, wie es Marianne van Kerkhoven einmal formulierte:,dramaturgy is a way of rehearsing collectivity“ (zit. nach MAAR 2020:181).

3. Ich halte es für elementar im Theater körperbewusst zu denken, auch oder gerade dann, wenn man sich für Sprache interessiert - die in Zeiten sozialer Distanz neu zu bewegen/berühren vermag.

Mich interessiert, wie der „Sauerstoff des Textes“ (GOSCH, zit. nach PETERS 2006:22) immer wieder in die Körper der Schauspieler*innen kommt, um dabei einen Stoffwechsel anzustiften, der ebenso spielerisch wie dramaturgisch faszinieren kann.

Thomas Köck weist genau auf diese Spannung hin:

Das ist nämlich das Interessante am Theater, dass dort Sprache auf Körper trifft. Oft vergisst man das ja. Man spürt dieses momentane, radikal gegenwärtige Aufeinandertreffen nicht mehr. Dieser Moment, in dem eine verdichtete Sprache in ihrer Seltsamkeit und Fremdheit mit einem sich vielleicht weigernden Körper kollidiert. (zit. nach STEINBUCH 2020:85)

4. „Es liegt zumeist mehr Kraft in einer Frage als in einer Antwort“ (NOETH 2020:285). Damit liegt das Ziel eher darin, Fragen weiter zu befragen und dabei groß genug, experimentell und möglichst radikal zu denken, d.h., aus der Gegenwart heraus Zukunft zu denken.

Dabei liegen die Dramaturgien auch in der künstlerischen Ausbildungspraxis nicht parat. Es gilt sie zusammen zu entwerfen, auszuprobieren, zu verwerfen und kontinuierlich neu/anders zu denken.

5. Ich halte an folgendem Leitgedanken, der insbesondere die schauspielerische Ausbildungspraxis betrifft, fest, da dieser auf die Anstiftung des Spielens abzielt, weswegen ich immer wieder gerne ins Theater gehe: „Theater ist ein Ort um neue/andere Sichtweisen auf das menschliche Zusammenleben zu erproben und die Welt darzustellen, wie sie ist oder sein könnte“ (UNIVERSITÄT GRAZ 2021).

Es geht um menschliche Konflikte, die auf der Bühne von Schauspieler*innen ebenso stellvertretend wie extrem direkt ausgehandelt werden. Allein in dieser Hinsicht wäre die Liquidation des Repräsentationsmodells fatal. 
Nino Haratischwili knüpft daran an und weist noch auf einen anderen, mir zentralen Punkt hin: „Theater sollte dem vertrauen, was es kann, - wenn es gelingt, den Menschen in all seiner Widersprüchlichkeit, seiner Verletzlichkeit, in seiner Grausamkeit, in seiner Schönheit zu zeigen“ (zit. nach MÜLLERWESEMANN 2020:47).

6. Theater verhandelt Leben und damit dringliche gesellschaftliche Diskurse, die in künstlerischen Kontexten ebenso in Form von theoretischen Positionen zu diskutieren sind, damit diese möglichst auch die künstlerischen Suchbewegungen befruchten, z. B. in Form von szenischen Essays (vgl. ADORNO 1978:9-49 / LEHMANN 1999:203-205 / TigGES 2020:52-76 / Tigges 2021:223-243). Aber theoretisches Wissen ist dem körperlichen Wissen und den Erfahrungen der Schauspieler*innen nicht überlegen (vgl. RUHSAM 2020:183-192). Stattdessen geht es mir um relationale dramaturgische Ansätze/Positionen, d.h. um das Teilen und Mitteilen von Wahrnehmungen und daraus entspringenden Ideen/Konzepten. Voraussetzung dafür ist eine große Beweglichkeit, die durch InBeziehung-Setzungen, durch Kombinationen, Verflechtungen und über das Aushandeln von unterschiedlichen Formen des Wissens ihre Kraft entfaltet - damit sich schließlich das individuell und kollektiv Generierte in möglichst konzentrierter Form wie ein (unvollständiges) Puzzle zusammenfügt (vgl. RUHSAM 2020:183-192).

Bleibt diese Wirkung aus oder geraten Prozesse des Ausprobierens ins Stocken, scheitere ich als dramaturgisches ,outside eye', ,Moderator', ,Brückenbauer oder ,Bindestrich', wenn ich meine Rolle auf die eines Korrektivs reduziere (vgl. UMATHUM / DECK 2020).

7. Die folgende Frage ist nicht neu, gewinnt aber gegenwärtig (wieder) spürbar an Bedeutung. Die Gründe dafür sind vielfältig. Ein auch durch den Zeitgeist geprägtes frisches Diskursbewusstsein, das zugleich ein scharfes Schlaglicht auf kunst-/theaterimmanente strukturelle Ermüdungs- und Krisenerscheinungen wirft, aber auch die (zu) strikte Trennung von künstlerischem und nicht künstlerischem Handeln berührt: Wie lassen sich soziale Situationen im künstlerischen Rahmen entwerfen und befragen? Und wie kann Dramaturgie als gesellschaftliches Handeln oder Erzählen als soziale Performanz neue Spiel- und Denkund Handlungsräume eröffnen? (vgl. HERBORDT / MOHREN 2020:97-112).

Zeigt nicht gerade der wachsende Drang der Schauspiel-Studierenden zu einer präsenteren und persönlicheren Autorschaft auf der Bühne, die nicht nur in den fast schon explodierenden Stückentwicklungen, sondern ebenso in der Auseinandersetzung mit (zu überschreibenden) Figuren aus dem klassischen Kanon 
spürbar wird, dass hier auch neue dramaturgische Spielräume entstehen? Und damit auch zwingend neue dramaturgische Kompetenzen ins Spiel kommen?

Die zukünftigen Schauspieler*innen sollten sich schon ihrer neuen Rollen als Co-Dramaturg*innen bewusst sein, oder?

Herausgefordert sind natürlich auch die zeitgenössischen Theaterautoren*innen selbstbewusst eigenwillige Spielvorlagen zu entwickeln und sich damit nicht den Stift aus der Hand nehmen zu lassen. Denn darauf wird es trotz aller ästhetischer/(post-)dramatischer Transformationen in Zukunft ebenso ankommen: Texte, die weiterhin bewusst für die Theaterpraxis geschrieben werden und sich durch ihre szenische Widerständigkeit auszeichnen.

Inwieweit diese, in bewusster Reibung zur Bühne geschriebenen Stücke längerfristig noch einen spürbaren Grad an ästhetischer Autonomie anstreben, die durch eine signifikante Autorschaft abgesichert ist und ob diese Stücke weiterhin gedruckt werden, ist eine andere Frage.

\section{Literatur}

Adorno, THEOdOR W. (1978): Noten zur Literatur I. Frankfurt a.M., 9-49.

Bourriaud, Nicolas (1998): Relational Aesthetics. Paris.

DECKER, GunNAR (2020): Komm, grosser Wind. Wolfram Lotz. In: Stückwerk, Arbeitsbuch Theater der Zeit. Berlin, 85.

Deuflhard, Amelie (2020): Gesellschaft neu denken. In: Theater heute. Jahrbuch. Berlin, 47.

Engler, Wolfgang (2017): Authentizität! Von Exzentrikern, Dealern und Spielverderbern. Berlin.

Fourest, CAROLE (2020): Generation Beleidigt. Von der Sprachpolizei zur Gedankenpolizei. Über den wachsenden Einfluss linker Identitärer. Berlin.

GÜRK, CHRISTOPH (2020): Der große Hack. Corona als Brandbeschleuniger der Digitalisierung - und als Angriff auf Staat und Gemeinschaft: Das Medium ist nie neutral. In: Theater heute. Jahrbuch. Berlin, 17.

HAYNER, JAKOB (2020): Warum Theater. Krise und Erneuerung. Berlin.

Herbordt, Bernhard / Mohren, Melanie (2020): Dramaturgie als gesellschaftliches Handeln. In: UMATHUM, SANDRA / DeCK, JAN (eds.): Postdramaturgien. Berlin, 97-112. HinRICHS, FABIAN (2018): Laudatio für Benny Claessens zur Verleihung des Alfred Kerr-Darstellerpreises, Berliner Festspiele v. 21.05.2018.

JESS, CAREN (2020): Box in der Box in der Box. In: Theater heute. Jahrbuch. Berlin, 53. 
KASCH, GeORG (2020): Echte Hosenrollen - Warum Schauspielerinnen heute so oft Männerrollen spielen. Die Ära der Frauen. In: Nachtkritik: www.nachtkritik.de/index.php?option=com_content\&view=article\&id=17596:hosenrollen-warum-schauspielerinnenheute-so-oft-maennerrollen-spielen\&catid=101\&Itemid=84 (23.01.2020).

KIRSCHNER, FRIEDRICH (2020): Teilhabe als Notwendigkeit: Theater als Raum pluraler Gemeinschaften. In: HEINRICH-BÖLL-STIFTUNG / NACHTKRITIK.DE IN ZUSAMMENARBEIT mit weltuebergang.Net / Diesselhorst, Sophie / HÜtter, Christiane / RaKow, Christian / Römer, Christian (eds.): Netztheater. Positionen, Praxis, Produktionen. Heinrich-Böll-Stiftung. Schriften zu Bildung und Kultur 14. Berlin, 70-73.

KLaeui, Andreas (2020): Von Null zur Autorschaft. Die Regisseurin Leonie Böhm sucht nach Menschen, die sich öffnen. In: Theater heute. Jahrbuch. Berlin, 105.

KÜMmel, Peter: Was uns alles so in den Knochen steckt. In: Die Zeit v. 10.09.2020:57.

LeHMAnN, HANs-Thies 1999: Postdramatisches Theater. Frankfurt a.M.

Lobbes, Marcus / Simon, Mario (2020): „Corona war ein Power-Up!“ Das erste Jahr der Dortmunder Akademie für Theater und Digitalität. Marcus Lobbes und Mario Simon im Interview. In: HEINRICH-BÖLL-STIFTUNG / NACHTKRITIK.DE In ZUSAMMENARBEIT mit weltuebergang.net / Diesselhorst, Sophie / Hütter, Christiane / Rakow, Christian / Römer, Christian (eds.): Netztheater. Positionen, Praxis, Produktionen. Heinrich-Böll-Stiftung. Schriften zu Bildung und Kultur 14. Berlin, 63-69.

MaAR, Kirsten (2020): P.S. - Postscriptum zur Post-Dramaturgie. In: Umathum, SANDRA / DeCK, JAN (eds.): Postdramaturgien. Berlin, 165-181.

Malzacher, Florian (2020): Gesellschaftsspiele. Politisches Theater heute. Berlin.

Müller-Schöll, Nikolaus (2020): Polizeiliche und politische Dramaturgie. In: Umathum, SANDRA / Deck, JAN (eds.): Postdramaturgien. Berlin, 209-230.

MÜLLER-WeSEMANN, BARBARA (2020): Aus der Sicherheit heraus entsteht keine Kunst. Nino Haratischwili. In: Stückwerk, Arbeitsbuch Theater der Zeit. Berlin, 47.

Noeth, SANDRA (2020): Notizen zu einem dramaturgischen Handlungsbegriff. In: Umathum, SANDRA / Deck, Jan (eds.): Postdramaturgien. Berlin, 280-286.

OSTERMEIER, THOMAS (2020): Reflexion und Abwägen. Muss man immer alles möglich machen? Über die Sehnsucht, sich wieder verstärkt in die Stoffe zu graben. In: Theater heute. Jahrbuch. Berlin, 53.

Peters, Nina (2006): Mit Beckett auf dem Abstellgleis. Der Regisseur Jürgen Gosch über Natürlichkeit, Scham und den Sauerstoff des Textes. In: Theater der Zeit 5:21-26.

Recke, Anta Helena (2019): Die Konstruktion von Whiteness im Theater. Im Gespräch mit Modelina Moka. In: RosaMag: https://rosa-mag.de/anta-helena-reckethematisiert-critical-whiteness-im-theater/ (15.09.2019).

REICHERT, Florian (2021): Präludien. In: SCHUBERT, FrAnK/Wigger, MARTIN (eds.): Wer bin ich, wenn ich spiele? Fragen an eine moderne Schauspielausbildung. Berlin, 13-17.

Ruhsam, Martina (2020): Dramaturgie der (und als) Kollaboration. In: Umathum, SANDRA / DeCK, JAN (eds.): Postdramaturgien. Berlin, 183-192. 


\section{Stefan Tigges}

Schäfer, Helmut (2020): Philosophisches Theater. Der Glanz des Scheins und das Elend des Dokuments. Frank M. Raddatz im Gespräch mit Helmut Schäfer. In: Lettre International 131:92-97.

SCHLOSSTHEATERS MOERS (2020): www.schlosstheater-moers.de/produktion/die-mutteraller-fragen-oder-25-rollen-die-eine-frau-niemals-spielen-sollte-premiere/ (15.09.2020).

SimONS, JoHAN (2018): Ich liebe das Nicht-Vollkommene. Johan Simons über Hamlet. In: Hamlet, Programmheft, Regie: Johan Simons, Premiere: 15.6.2019, Schauspielhaus Bochum, 15-18.

Simons, JoHAn (2019). Programmheft Hamlet. Schauspielhaus Bochum, Spielzeit 2019/2020, 15.

SimOns, JoHAN (2020): Ein Turm zu Babel. In: Theater heute. Jahrbuch. Berlin, 2018, $70 f$.

SQUAD, GoB (2020): Meet the opposite. In: Theater heute. Jahrbuch. Berlin, 60-62.

Steinbuch, Gerhild (2020): Hinter den Räumen. In: UMATHUM, SANDRA / Deck, JAN (eds.): Postdramaturgien. Berlin, 84-95.

StEMANN, Nicolas (2019): Agenten für Diversität. In: Theater heute. Jahrbuch. Berlin, 63-72.

Tigges, Stefan (2020): Stückentwicklungen. Szenische Diskurspotentiale und dramaturgische Transformationen. In: NISSEN-RIZVANI, KARIN / SCHÄFER, MARTIN-JÖRG (eds.): TogetherText. Prozessual erzeugte Texte im Gegenwartstheater, Theater der Zeit, Recherchen 155. Berlin, 52-76.

Tigges, Stefan (2021): Der diskrete Charme des Flüchtigen. Thom Luz’ Stückentwicklung Girl from the fog machine factory. In: FAMULA, MARTA / WiTSCHEL, VERENA (eds.): Theater und Krise. Paradigmen der Störung in Dramentexten und Bühnenkonzepten nach 2000. Paderborn, 223-243.

Umathum, SANDra / Deck, JAN (eds.) (2020): Postdramaturgien. Berlin.

UnIVERSITÄt GraZ (2021): Homepage des Studiengangs Schauspiel: https://schauspiel.kug.ac.at (13.05.2021).

WiLle, Franz (2006): Sein vor Sinn. In: Theater heute. Jahrbuch. Berlin, 114.

WiLle, FranZ (2019): Was ist da eigentlich passiert? Wichtige Entwicklungen im Theater der 10er Jahre und darüber hinaus - am Beispiel einiger markanter Inszenierungen. In: Theater heute. Jahrbuch. Berlin, 104.

Zellner, Juliane / LoBBes, MARCus / ZiPf, JonAs (eds.) (2021): transformers. digitalität. inklusion. nachhaltigkeit. In: Theater der Zeit. Arbeitsbuch. Berlin, 100-107, 120$125,129-132$. 